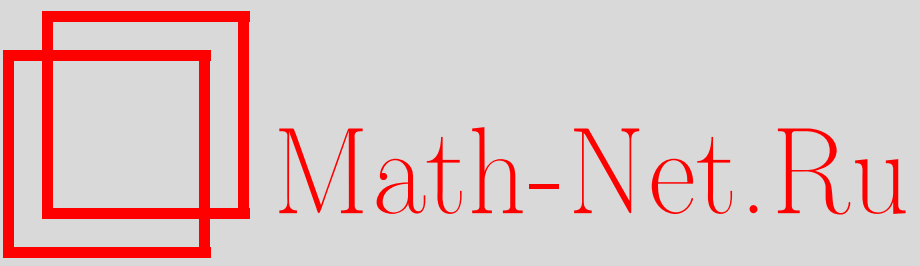

В. П. Казанцев, Вариационные принципы, электрические характеристические мультиполи и высшие поляризуемости в теории поля, ТМФ, 1999, том 119, номер 3, 441-454

DOI: https://doi.org/10.4213/tmf751

Использование Общероссийского математического портала Math-Net.Ru подразумевает, что вы прочитали и согласны с пользовательским соглашением

http: //www . mathnet.ru/rus/agreement

Параметры загрузки:

IP : 54.205 .225 .156

26 апреля 2023 г., 12:22:05 


\section{ВАРИАЦИОННЫЕ ПРИНЦИПЫ, ЭЛЕКТРИЧЕСКИЕ ХАРАКТЕРИСТИЧЕСКИЕ МУЛЬТИПОЛИ И ВЫСШИЕ ПОЛЯРИЗУЕМОСТИ В ТЕОРИИ ПОЛЯ}

На основе вариационных принципов введены новые понятия о характеристических электрических мультиполях, представляющих собой систему базисных распределений зарядов по границе области пространства. Потенциалы характеристических мультиполей внутри рассматриваемой области суть гармонические полиномы, степени которых определяют минимальный порядок отличного от нуля сферического мультипольного момента характеристического мультиполя. С помощью формализма характеристических мультиполей решена проблема моментов в электростатике, построен лагранжиан сверхпроводника в электростатическом поле. Через потенциалы характеристических мультиполей выражена функция Грина для уравнения Лапласа в свободном пространстве.

\section{1. ВВЕДЕНИЕ}

Мультипольным моментом ограниченной в пространстве системы зарядов называют совокупность из $2 l+1$ величин [1], определяемых с помошью интеграла

$$
q_{l m}=\int r^{l} y_{l m}(\theta, \alpha) d q(r)
$$

в котором $\vec{r}$ - радиус-вектор точки пространства; $(r ; \theta ; \alpha)$ - его сферические координаты; $y_{l m}(\theta, \alpha)$ - вешественные функшии, выражаемые через сферические гармоники [2] $Y_{l m}(\theta, \alpha)$ по формулам

$$
y_{l m}(\theta, \alpha)=\sqrt{\frac{8 \pi}{2 l+1}} \begin{cases}\frac{1}{2}\left((-1)^{m} Y_{l m}(\theta, \alpha)+Y_{l,-m}(\theta, \alpha)\right), & m>0, \\ \frac{1}{\sqrt{2}} Y_{l 0}(\theta, \alpha), & m=0, \\ \frac{i}{2}\left(Y_{l m}(\theta, \alpha)-(-1)^{m} Y_{l,-m}(\theta, \alpha)\right), & m<0 .\end{cases}
$$

*Красноярский государственный университет, Красноярск, Россия. E-mail: kazantc.@phys.kgu.krasnoyarsk.su 
Разложение по мультиполям потенциала некоторой ограниченной в пространстве системы зарядов

$$
\varphi(\vec{r})=\frac{1}{4 \pi \varepsilon_{0}} \int \frac{d q\left(\vec{r}^{\prime}\right)}{\left|\vec{r}-\vec{r}^{\prime}\right|}
$$

проводят с помошью соотношения

$$
\frac{1}{\left|\vec{r}-\vec{r}^{\prime}\right|}=\sum_{l=0}^{\infty} r^{\prime l} r^{l+1} \sum_{m=-l}^{l} y_{l m}(\theta, \alpha) y_{l m}\left(\theta^{\prime}, \alpha^{\prime}\right),
$$

справедливого при $r>r^{\prime}$. В результате приходят к формуле

$$
\varphi(\vec{r})=\frac{1}{4 \pi \varepsilon_{0}} \sum_{l=0}^{\infty} \frac{1}{r^{l+1}} \sum_{m=-l}^{l} q_{l m} y_{l m}(\theta, \alpha) .
$$

В случае, если заряды распределены по поверхности с поверхностной плотностью $\sigma(\vec{r})$, имеем

$$
d q(\vec{r})=\sigma(\vec{r}) d S
$$

где $d S$ - элемент плошади поверхности. Заметим, что $q_{l m}$ инвариантны относительно параллельного переноса системы координат, если все $q_{k n}$ равны нулю при $k<l$.

Утверждение о том, что из всех распределений зарядов по поверхности уединенного проводника, имеющих одинаковые $q_{00}$, минимумом электростатической энергии обладает истинное распределение, носит название принципа Гаусса [3]. Иначе это утверждение можно рассматривать как одну из вариационных формулировок задачи о емкости проводника.

Для распределений зарядов по поверхности проводника с $q_{00}=0$ и постоянными $q_{1 \mathrm{~m}}$ минимум электростатической энергии достигается на решении задачи о поляризуемости проводника в однородном электрическом поле [4]. Естественным образом возникает задача о минимуме электростатической энергии для распределений зарядов по поверхности с $q_{k m}=0$ при $k<l$ и постоянных $q_{l m}$. Интересна будет и физическая интерпретация такой задачи, центральное место в которой будут занимать некоторые базисные распределения зарядов по поверхности проводника. Мы будем называть эти распределения характеристическими мультиполями поверхности $\partial V$. Введение и исследование понятий характеристических мультиполей - основная цель настояшей работы.

\section{2. ПОНЯТИЯ О ХАРАКТЕРИСТИЧЕСКИХ МУЛЬТИПОЛЯХ}

Распределениям зарядов $\sigma(\vec{r})$ по поверхности $\partial V$ соответствуют потенциалы

$$
\varphi(\vec{r})=\frac{1}{4 \pi \varepsilon_{0}} \int_{\partial V} \frac{\sigma\left(\vec{r}^{\prime}\right)}{\left|\vec{r}-\vec{r}^{\prime}\right|} d S^{\prime}
$$

$\mathrm{K}$ допустимым $\sigma(\vec{r})$ отнесем такие, что

$$
\int_{\partial V} \sigma(r) r^{k} y_{k n}(\theta, \alpha) d S=q_{l m} \delta_{k l} \delta_{m n}, \quad k \leqslant l,
$$


где $\delta_{i j}-$ символ Кронекера. Ищем минимум функционала энергии

$$
W(\varphi)=\frac{\varepsilon_{0}}{2} \int_{\Omega}(\nabla \varphi)^{2} d V
$$

Здесь интеграл берется по объему области всего пространства $\Omega$.

Рассмотрим непосредственно проверяемое тождество

$$
W(\varphi)=W\left(\varphi_{0}\right)+W\left(\varphi-\varphi_{0}\right)+\varepsilon_{0} \int_{\Omega} \nabla \varphi_{0} \cdot \nabla\left(\varphi-\varphi_{0}\right) d V
$$

интеграл $I$ в правой части которого может быть преобразован к виду

$$
I=\int_{\partial V} \varphi_{0} \cdot\left(\sigma-\sigma_{0}\right) d S
$$

Здесь $\sigma$ и $\sigma_{0}$ - распределения зарядов по поверхности $\partial V$, отвечаюшие согласно (2) потенциалам $\varphi$ и $\varphi_{0}$. Интеграл $I$ обрашается в нуль, если $\varphi_{0}$ принимает на $\partial V$ значение

$$
\left.\varphi_{0}\right|_{\partial V}=\sum_{k=0}^{l} r^{k} \sum_{n=-k}^{k} a_{k n} y_{k n}(\theta, \alpha)
$$

где $a_{k n}$ - постоянные. В этом легко убедиться, обратившись к условиям (3), которым удовлетворяют как $\sigma$, так и $\sigma_{0}$. Тогда из тождества (5) следует

$$
W(\varphi)-W\left(\varphi_{0}\right)=W\left(\varphi-\varphi_{0}\right)>0
$$

что и доказывает минимальность $W(\varphi)$ на $\varphi_{0}$.

В единственности минимизируюшей функционал (4) функции $\varphi_{0}$ можно убедиться на основании соотношения (7), записав

$$
W\left(\varphi_{0}\right)-W\left(\varphi_{0}^{\prime}\right)=W\left(\varphi_{0}-\varphi_{0}^{\prime}\right)=W\left(\varphi_{0}^{\prime}\right)-W\left(\varphi_{0}\right),
$$

где $\varphi_{0}$ и $\varphi_{0}^{\prime}$ - два потенциала, удовлетворяющих условиям (3) и (6) с возможно различными постоянными $a_{k n}$ для $\varphi_{0}$ и $\varphi_{0}^{\prime}$. Соотношения (8) будут, очевидно, иметь место, если $W\left(\varphi_{0}-\varphi_{0}^{\prime}\right)=0$, т.е. если $\nabla \varphi_{0}=\nabla \varphi_{0}^{\prime}$ или $\varphi_{0}=\varphi_{0}^{\prime}$, поскольку допустимые потенциалы согласно (2) нормированы на нуль в бесконечно удаленной точке.

Из единственности $\varphi_{0}$ следует единственность $a_{k n}$ в условии (6). Вследствие линейности рассматриваемой задачи $a_{k n}$ должны линейно зависеть от $\chi_{l m}$, обрашаясь в нуль при $\chi_{l m}=0$. Поэтому, в частности, можно записать

$$
a_{l n}=\beta_{n m}^{(l)} \chi_{l m} .
$$


Можно считать, что $m$ и $n$ независимо пробегают целые значения из интервала $[-l ; l]$. Коэффициенты $\beta_{n m}^{(l)}$ составляют матрицу размерности $(2 l+1) \times(2 l+1)$. Чтобы исследовать свойства этой матрищы, введем обозначения $\chi_{l m} \varphi_{l m}$ для потенциала, минимизируюшего функционал (4) при условии $(3)$, и $\chi_{l m} \sigma_{l m}-$ соответствуюшую поверхностную плотность зарядов. Подставим в функционал $W(\varphi)$ величину

$$
\varphi=\sum_{m=-l}^{l} \chi_{l m} \varphi_{l m}
$$

В результате такой подстановки получим

$$
\begin{gathered}
W(\varphi)=\frac{1}{2} \vec{\chi}_{l} \cdot \widehat{B}^{(l)} \cdot \vec{\chi}_{l}, \\
\vec{\chi}_{l}=\left(\chi_{l,-l} ; \ldots ; \chi_{l l}\right) .
\end{gathered}
$$

Элементы матрищы $\widehat{B}^{(l)}$ находим следуюшим образом:

$$
B_{n m}^{(l)}=\varepsilon_{0} \int_{\Omega} \nabla \varphi_{l n} \cdot \nabla \varphi_{l m} d V
$$

следовательно, $\widehat{B}$ - симметричная матрица, а из положительности $W(\varphi)$ следует положительная определенность $\widehat{B}$. Выражение (9) можно также преобразовать к виду

$$
B_{n m}^{(l)}=\int_{\partial V} \sigma_{l n} \varphi_{l m} d S=\int_{\partial V} \sigma_{l m} \varphi_{l n} d S=\beta_{n m}^{(l)}=\beta_{m n}^{(l)} .
$$

Укажем, что $\varphi_{0}$ минимизирует функционал

$$
L(\varphi)=W(\varphi)-\chi_{l m} a_{l m}
$$

на классе непрерывных кусочно-гладких потенциалов, принимающих на $\partial V$ значения (6). Из условия (6) следует, что $\varphi_{0}(\vec{r})$ есть линейная комбинация потенциалов $\psi_{l m}(\vec{r})$ - решений уравнения Лапласа вне $\partial V$ с граничными условиями

$$
\left.\psi_{l m}(\vec{r})\right|_{\partial V}=r^{l} y_{l m}(\theta, \alpha)
$$

Сушествование $\psi_{l m}(\vec{r})$ будет обеспечивать и существование $\varphi_{0}(\vec{r})$. Процесс ортогонализации системы функций $\psi_{l m}(\vec{r})$ с различными $l$ приведет к построению базиса потенциалов, сопряженного базису потенциалов характеристических мультиполей $\varphi_{l m}(\vec{r})$.

Заметим, что функции $\varphi_{l_{1} m_{1}}$ и $\varphi_{l_{2} m_{2}}$ с различными $l_{1}$ и $l_{2}$ ортогональны в том смысле, что

$$
\varepsilon_{0} \int_{\Omega} \nabla \varphi_{l_{1} m_{1}} \cdot \nabla \varphi_{l_{2} m_{2}} d V=\beta_{m_{1} m_{2}}^{(l)} \delta_{l_{1} l_{2}} .
$$

Интересно, что функции $\chi_{l m} \varphi_{l m}(\vec{r})$, как это видно из граничного для них условия $(6)$, в области $V$ представляют собой гармонические полиномы степени $l$. Их выражение 
будет совпадать с правой частью граничного условия (6). В области $V \chi_{l m} \nabla \varphi_{l m}$ можно интерпретировать как напряженность электрического поля, создаваемого внешними источниками в области проводника. В связи с этим исследуемая задача позволяет ответить на вопрос, какое внешнее поле необходимо создать в области $V$ в отсутствие проводника, чтобы при его внесении в это поле в результате его поляризации все мультипольные моменты проводника с $k \leqslant l$ были равны нулю, кроме $\chi_{l m}$. Параметры $a_{l n}$ тогда будут определять постоянные значения производных $l$-го порядка от потенциала внешнего поля в области $V$. Допуская теперь, что они могут изменяться произвольным образом, значения соответствующих им мультипольных моментов $\vec{\chi}_{l}$ найдем по формуле

$$
\vec{\chi}_{l}=\left(\hat{\beta}^{(l)}\right)^{-1} \cdot \vec{a}_{l}=\hat{\alpha}^{(l)} \cdot \vec{a}_{l}
$$

Матрицу, которая связывает постоянные $a_{l n}$, характеризующие внешнее электрическое поле, с мультиполями $\chi_{l n}$, наведенными согласно данной выше интерпретации рассматриваемой задачи на проводнике внешним полем, по аналогии с задачей о поляризуемости проводника в однородном электрическом поле [5] естественно назвать матрицей поляризуемости $l$-го порядка. Все такие матрищы положительно-определены и симметричны. Через них может быть выражена электростатическая энергия поляризационных зарядов:

$$
W(\varphi)=\frac{1}{2} \vec{a}_{l} \cdot \hat{\alpha}^{(l)} \cdot \vec{a}_{l}
$$

Таким образом, каждая поверхность $\partial V$ характеризуется системой положительноопределенных симметричных матриц $\hat{\alpha}^{(l)}$, а также связанными с этими матрицами системой из $2 l+1$ потенциалов $\varphi_{l m}(\vec{r})$ и соответствующими этим потенциалам распределениями зарядов $\sigma_{l m}(\vec{r})$ по поверхности $\partial V$. Эти распределения естественно называть характеристическими мультиполями поверхности $\partial V$.

\section{3. ХАРАКТЕРИСТИЧЕСКИЕ МУЛЬТИПОЛИ И ВЫСШИЕ ПОЛЯРИЗУЕМОСТИ ДЛЯ ШАРА}

Для областей сложной формы задача определения $\hat{\alpha}^{(l)}, \varphi_{l m}(\vec{r})$ и $\sigma_{l m}(\vec{r})$ весьма сложна, поскольку даже $\hat{\alpha}^{(0)}$ (емкость проводника) известна лишь для чуть более десятка различных форм проводников [6], большая часть из которых представляет собой различные модификации эллипсоида. Поляризуемость проводника $\hat{\alpha}^{(1)}$ известна лишш для эллипсоида и его модификаций [5]. Поляризуемости более высокого порядка чем 1 в теоретической и математической физике до сих пор не рассматривались.

Относительно просто $\hat{\alpha}^{(l)}, \varphi_{l m}(\vec{r})$ и $\sigma_{l m}(\vec{r})$ могут быть построены для шара. Приведем сразу результаты:

$$
\begin{aligned}
& \varphi_{l m}(\vec{r})=\frac{1}{4 \pi \varepsilon_{0}} y_{l n}(\theta, \alpha) \begin{cases}r^{-(l+1)}, & r \geqslant R, \\
\frac{r^{l}}{R^{2 l+1}}, & r \leqslant R,\end{cases} \\
& \sigma_{l m}(\vec{r})=\frac{(2 l+1) y_{l m}(\theta, \alpha)}{4 \pi R^{l+2}}, \quad \hat{\alpha}^{(l)}=4 \pi \varepsilon_{0} R^{2 l+1} \hat{e}_{l},
\end{aligned}
$$


где начало системы координат совпадает с центром шара радиуса $R, \hat{e}_{l}$ - единичная матрица размерности $(2 l+1) \times(2 l+1)$.

Характеристические мультиполи и высшие поляризуемости для эллипсоидов врашения построены в работе [7].

\section{4. ПРИМЕРЫ ХАРАКТЕРИСТИЧЕСКИХ МУЛЬТИПОЛЕЙ ДЛЯ РЕШЕНИЯ ЗАДАЧ ЭЛЕКТРОСТАТИКИ}

Рассмотрим задачу о проводнике, находящемся во внешнем электрическом поле. Если проводник помешен во внешнее электрическое поле с потенциалом $U(\vec{r})$, то заряды по его поверхности перемешаются так, что создаваемое ими поле компенсирует внешнее поле в области проводника. На поверхности проводника заряды создадут электрический потенциал, отличающийся от $-U(\vec{r})$ не более чем на постоянную. Решение соответствуюшей краевой задачи о поле поляризационных зарядов в случае, если проводник не заряжен, можно искать как сумму мультиполей

$$
\varphi(\vec{r})=\sum_{l=1}^{\infty} \vec{\chi}_{l} \cdot \vec{\varphi}_{l}(\vec{r}),
$$

минимизирующую функционал

$$
L(\varphi)=W(\varphi)+\int_{\partial V} \sigma U d S
$$

Как было показано в предыдушем разделе, для пробной функции вида (10) имеем

$$
W(\varphi)=\frac{1}{2} \sum_{l=1}^{\infty} \vec{\chi}_{l} \cdot \hat{\beta}^{(l)} \cdot \vec{\chi}_{l}, \quad \sigma=\sum_{l=1}^{\infty} \vec{\chi}_{l} \cdot \vec{\sigma}_{l} .
$$

Минимизируя функционал (11) по $\vec{\chi}_{l}$, найдем

$$
\vec{\chi}_{l}=-\hat{\alpha}^{(l)} \cdot \int_{\partial V} \vec{\sigma}_{l} U d S
$$

и

$$
\varphi(\vec{r})=-\sum_{l=1}^{\infty} \int_{\partial V} \vec{\sigma}_{l}\left(\vec{r}^{\prime}\right) U\left(\vec{r}^{\prime}\right) d S^{\prime} \cdot \hat{\alpha}^{(l)} \cdot \vec{\varphi}_{l}(\vec{r}) .
$$

Соответствующая потенциалу (12) плотность зарядов на границе проводника может быть получена с помощью соотношения

$$
\sigma(\vec{r})=-\sum_{l=1}^{\infty} \int_{\partial V} \vec{\sigma}_{l}\left(\vec{r}^{\prime}\right) U\left(\vec{r}^{\prime}\right) d S^{\prime} \cdot \hat{\alpha}^{(l)} \cdot \vec{\sigma}_{l}(\vec{r})
$$

Если же проводник имеет заряд $q=\chi_{00}$, то к сумме (12) нужно будет добавить слагаемое $q \varphi_{00}$, а к плотности зарядов $(13)$ - слагаемое $q \sigma_{00}$. 
Таким образом, использование аппарата высших поляризуемостей позволяет получать решение основной задачи электростатики для проводника во внешнем электрическом поле в виде бесконечных рядов по функциям $\varphi_{l m}(\vec{r})$ и $\sigma_{l m}(\vec{r})$.

Потенциал зарядов, распределенных по поверхности с плотностью $\sigma(\vec{r})$, можно найти, минимизируя функционал

$$
L(\varphi)=W(\varphi)-\int_{\partial V} \sigma \varphi d S .
$$

Потенциал $\varphi(\vec{r})$ теперь ишем в виде

$$
\varphi(\vec{r})=\sum_{l=0}^{\infty} \vec{\chi}_{l} \cdot \varphi_{l}
$$

После подстановки потенциала $\varphi(\vec{r})$ в функционал $(14)$, получим

$$
L(\varphi)=\frac{1}{2} \sum_{l=0}^{\infty} \vec{\chi}_{l} \cdot \hat{\beta}^{(l)} \cdot \vec{\chi}_{l}-\sum_{l=0}^{\infty} \vec{\chi}_{l} \cdot \int_{\partial V} \vec{\varphi}_{l}(\vec{r}) \vec{\sigma}(\vec{r}) d S .
$$

Минимизируя полученное значение функционала по $\vec{\chi}_{l}$, найдем

$$
\begin{aligned}
\vec{\chi}_{l} & =\hat{\alpha}^{(l)} \cdot \int_{\partial V} \vec{\varphi}_{l}(\vec{r}) \vec{\sigma}(\vec{r}) d S, \\
\varphi(\vec{r}) & =\sum_{l=0}^{\infty} \int_{\partial V} \vec{\varphi}_{l}\left(\vec{r}^{\prime}\right) \vec{\sigma}\left(\vec{r}^{\prime}\right) d S^{\prime} \cdot \hat{\alpha}^{(l)} \cdot \vec{\varphi}_{l}(\vec{r}) .
\end{aligned}
$$

\section{5. МУЛЬТИПОЛЬНЫЕ РАЗЛОЖЕНИЯ}

Соотношения (16) можно интерпретировать как разложение по потенциалам $\varphi_{\operatorname{lm}}(\vec{r})$ характеристических мультиполей $\sigma_{l m}(\vec{r})$ для потенциала, создаваемого зарядами, расположенными на поверхности $\partial V$. Для энергии таких зарядов будет справедлива формула

$$
W(\varphi)=\frac{1}{2} \sum_{l=0}^{\infty} \vec{\chi}_{l} \cdot \hat{\beta}^{(l)} \cdot \vec{\chi}_{l},
$$

следующая из условия ортогональности потенциалов мультиполей (9) и представляющая собой мультипольное разложение электростатической энергии поверхностных зарядов.

Рассмотрим теперь задачу разложения по мультиполям потенциала

$$
\varphi(\vec{r})=\frac{1}{4 \pi \varepsilon_{0}} \int_{V} \frac{d q\left(\vec{r}^{\prime}\right)}{\left|\vec{r}-\vec{r}^{\prime}\right|}
$$

создаваемого некоторой системой зарядов, лежаших внутри поверхности $\partial V$. Представим потенциал (18) в области $\Omega-V$ суммой потенциалов мультиполей

$$
\varphi(\vec{r})=\sum_{l=0}^{\infty} \vec{\chi}_{l} \cdot \vec{\varphi}_{l}(\vec{r})
$$


Точно так же, как и при решении задачи о проводнике, помешенном во внешнее электрическое поле, найдем

$$
\vec{\chi}_{l}=\hat{\alpha}^{(l)} \cdot \int_{\partial V} \varphi(\vec{r}) \sigma_{l}(\vec{r}) d S
$$

Подставляя $\varphi(\vec{r})$ из соотношения $(18)$ в эту формулу и учитывая, что

$$
\vec{\varphi}_{l}\left(\vec{r}^{\prime}\right)=\frac{1}{4 \pi \varepsilon_{0}} \int_{\partial V} \frac{\vec{\sigma}_{l}(\vec{r})}{\left|\vec{r}-\vec{r}^{\prime}\right|} d S
$$

получим

$$
\vec{\chi}_{l}=\hat{\alpha}^{(l)} \cdot \int_{V} \vec{\varphi}_{l}(\vec{r}) d q(\vec{r}) .
$$

Эта формула вместе с формулой (19) описьвает мультипольное разложение потенциала $(18)$ в области $\Omega-V$.

Соотношение (19) можно записать как

$$
\varphi(\vec{r})=\sum_{l=0}^{\infty} \int_{V} \vec{\varphi}_{l}\left(\vec{r}^{\prime}\right) \cdot \hat{\alpha}^{(l)} \cdot \vec{\varphi}_{l}(\vec{r}) d q\left(\vec{r}^{\prime}\right) .
$$

Сравнивая два представления потенциала (18) и (22), приходим к следующему разложению функции Грина оператора Лапласа в $R^{3}$ :

$$
\sum_{l=0}^{\infty} \vec{\varphi}_{l}\left(\vec{r}^{\prime}\right) \cdot \hat{\alpha}^{(l)} \cdot \vec{\varphi}_{l}(\vec{r})=\frac{1}{4 \pi \varepsilon_{0}} \frac{1}{\left|\vec{r}-\vec{r}^{\prime}\right|}, \quad \vec{r} \in \Omega-V, \quad \vec{r}^{\prime} \in V
$$

позволяющему разложить по потенциалам мультиполей $\varphi_{l m}(\vec{r})$ поле произвольной системы зарядов, заключенной в области $V$. В частности, когда $V$-шар, соотношение (23) переходит в известное разложение (1) функции Грина оператора Лапласа по сферическим мультиполям.

Таким образом, приходим к выводу, что каждая замкнутая поверхность $\partial V$ порождает свою систему мультиполей, разложение по которым потенциала зарядов, содержащихся внутри $\partial V$, будет иметь место во всей области $\Omega-V$.

Чтобы найти плотность поверхностных зарядов на $\partial V$, создаюших в области $\Omega-V$ такой же потенциал $(18)$, как и распределение зарядов $d q(\vec{r})$ по области $V$ (задача выметания Пуанкаре [8]), следует воспользоваться соотношением

$$
\sigma(\vec{r})=\sum_{l=0}^{\infty} \vec{\chi}_{l} \cdot \vec{\sigma}_{l}(\vec{r})
$$

в котором мультипольный момент $\vec{\chi}_{l}$ определен формулой (21).

Соответствующая плотности поверхностных зарядов (24) электростатическая энергия (17) обладает определенным свойством минимальности. Действительно, любое продолжение потенциала $\varphi(\vec{r})$ с поверхности $\partial V$ в область $V$ согласно принципу Дирихле [9] даст для электростатической энергии, локализованной в области $V$, значение 
большее, чем решение уравнения Лапласа, принимающее на $\partial V$ значения $\varphi(\vec{r})$ из (18). Следовательно, можно утверждать, что из всех распределений зарядов по $V \cup \partial V$, создаюших в области $\Omega-V$ одинаковые потенциалы $\varphi(r)$, минимальной электростатической энергией будут обладать заряды, распределенные на поверхности $\partial V$.

Аналогичным образом может быть рассмотрена задача о мультипольном разложении потенциала

$$
\varphi(\vec{r})=\frac{1}{4 \pi \varepsilon_{0}} \int_{\Omega-V} \frac{d Q\left(\vec{r}^{\prime}\right)}{\left|\vec{r}-\vec{r}^{\prime}\right|}
$$

зарядов $d Q(\vec{r})$, расположенных в области $\Omega-V$. При решении этой задачи проше всего непосредственно воспользоваться формулой (23), с помощью которой относительно просто можно записать мультипольное разложение потенциала (25) в области $V$ :

$$
\varphi(\vec{r})=\sum_{l=0}^{\infty} \int_{\Omega-V} \vec{\varphi}_{l}\left(\vec{r}^{\prime}\right) \cdot \hat{\alpha}^{(l)} \cdot \vec{\varphi}_{l}(\vec{r}) d Q\left(\vec{r}^{\prime}\right) .
$$

Для определения плотности поверхностных зарядов, создаюших в области $V$ такой же потенциал (25), что и распределение зарядов $d Q(\vec{r})$, можно воспользоваться формулой (24), куда вместо $\vec{\chi}_{l}$ следует подставить

$$
\vec{X}_{l}=\hat{\alpha}^{(l)} \cdot \int_{\Omega-V} \vec{\varphi}_{l}(\vec{r}) d Q(\vec{r}) .
$$

Тем самым решается задача о “выметании” зарядов из области $\Omega-V$ на поверхность $\partial V$. Соответствующая найденной таким образом $\sigma(\vec{r})$ электростатическая энергия, определяемая формулами (17) и (27), будет меньше электростатической энергии распределений зарядов $d Q(r)$, создаюших в области $V$ потенциал (25).

С помошью разложения функции Грина (23) можно представить энергию взаимодействия $W_{\text {int }}$ распределений зарядов $d q(\vec{r})$ в области $V$ и $d Q(\vec{r})$ в области $\Omega-V$ в виде мультипольного разложения

$$
W_{\mathrm{int}}=\frac{1}{4 \pi \varepsilon_{0}} \int_{V} \int_{\Omega-V} \frac{1}{\left|r-r^{\prime}\right|} d Q\left(r^{\prime}\right) d q(r)=\sum_{l=0}^{\infty} \vec{\chi}_{l} \cdot \hat{\beta}^{(l)} \cdot \vec{X}_{l} .
$$

Заметим, что в отличие от величин $\chi_{l m}$, которые естественно называть внутренними мультипольными моментами, $X_{l m}$ назовем внешними мультипольными моментами. В силу того что $\varphi(\vec{r})$ представляют собой непрерывные на $\partial V$ функции, для распределений зарядов по $\partial V$ получим $X_{l m}=\chi_{l m}$.

Функция

$$
G\left(\vec{r}, \vec{r}^{\prime}\right)=\frac{1}{4 \pi} \frac{1}{\left|\vec{r}-\vec{r}^{\prime}\right|}-\varepsilon_{0} \sum_{l=0}^{\infty} \vec{\varphi}_{l}\left(\vec{r}^{\prime}\right) \cdot \hat{\alpha}^{(l)} \cdot \vec{\varphi}_{l}(\vec{r})
$$

обращается в нуль, если хотя бы одна из точек $\vec{r}$ или $\vec{r}^{\prime}$ лежит на границе области $\partial V$. С другой стороны,

$$
\Delta G\left(\vec{r}, \vec{r}^{\prime}\right)=\Delta^{\prime} G\left(\vec{r}, \vec{r}^{\prime}\right)=-\delta\left(\vec{r}-\vec{r}^{\prime}\right),
$$

где $\delta\left(\vec{r}-\vec{r}^{\prime}\right)$ - дельта-функция Дирака. Таким образом, $G\left(\vec{r}, \vec{r}^{\prime}\right)$ будет служить функцией Грина задачи Дирихле для уравнения Лапласа как в области $V$, так и в области $\Omega-V$.

4 Теоретическая и математическая физика, т. 119, № 3, 1999 г. 


\section{6. ПРОБЛЕМА МОМЕНТОВ В ЭЛЕКТРОСТАТИКЕ}

Заметим, что согласно соотношению (21) характеристические мультипольные моменты удобно выражать с помошью базисных функций

$$
\Phi_{l m}(\vec{r})=\sum_{n=-l}^{l} \alpha_{m n} \varphi_{l n}(\vec{r})=r^{l} y_{l m}(\theta, \alpha)+\sum_{k=0}^{l-1} \sum_{p=-k}^{k} A_{k p}^{(l m)} r^{k} y_{k p}(\theta, \alpha)
$$

по формуле

$$
\chi_{l m}=\int_{V} \Phi_{l m}(\vec{r}) d q(\vec{r})=q_{l m}+\sum_{k=0}^{l-1} \sum_{p=-k}^{k} A_{k p}^{(l m)} q_{k p},
$$

где $q_{l m}$ - сферические мультипольные моменты системы зарядов.

Справедливость связи между характеристическими $\chi_{l m}$ и сферическими $q_{l m}$ мультипольными моментами:

$$
\chi_{l m}=\int_{V} \Phi_{l m}(\vec{r}) d q(\vec{r})=q_{l m}+\sum_{k=0}^{l-1} \sum_{p=-k}^{k} A_{k p}^{(l m)} q_{k p},
$$

не вызывает сомнения, если система зарядов $d q(\vec{r})$ расположена в области $V$. Если же система зарядов $d q(\vec{r})$ не лежит целиком в области $V$, то $q_{l m}$ по-прежнему будут иметь смысл сферических мультиполей, тогда как $\chi_{l m}$ уже не могут быть найдены по первой формуле (29) и, вообше говоря, теряют смысл характеристических мультипольных моментов. Тем не менее $\chi_{l m}$ можно определить формально на основе соотношения (30). Покажем, что тогда в окрестности бесконечно удаленной точки будет выполнено равенство

$$
\frac{1}{4 \pi \varepsilon_{0}} \sum_{l=0}^{\infty} \sum_{m=-l}^{l} q_{l m} \frac{y_{l m}(\theta, \alpha)}{r^{l+1}}=\sum_{l=0}^{\infty} \sum_{m=-l}^{l} \chi_{l m} \varphi_{l m}(\vec{r}) .
$$

В самом деле, потенциалу, определяемому конечным отрезком ряда левой части (31),

$$
\varphi^{(N)}(\vec{r})=\frac{1}{4 \pi \varepsilon_{0}} \sum_{l=0}^{N} \sum_{m=-l}^{l} q_{l m} \frac{y_{l m}(\theta, \alpha)}{r^{l+1}}=\sum_{l=0}^{\infty} \sum_{m=-l}^{l} \chi_{l m}^{(N)} \varphi_{l m}(\vec{r})
$$

отвечает распределение зарядов $d q^{(N)}(\vec{r})$, например, расположенных в области $V$ на сфере достаточно малого радиуса $R$ :

$$
d q^{(N)}(\vec{r})=\left(\sum_{l=0}^{N} \sum_{m=-l}^{l} q_{l m} \sigma_{l m}(\theta, \alpha)\right) \delta(r-R) d V .
$$

Распределению зарядов (33) будут соответствовать характеристические мультипольные моменты

$$
\chi_{l m}^{(N)}=\int_{V} \Phi_{l m}(\vec{r}) d q^{(N)}(\vec{r})
$$


для которых справедливы равенства

$$
\chi_{l m}^{(N)}=\chi_{l m} \quad \text { при } \quad l \leqslant N,
$$

где $\chi_{l m}$ определены формулой (30). Поскольку равенство (32) справедливо для любого $N$, то переходя в нем к пределу $N \rightarrow \infty$, учитывая при этом переходе соотношения (34) и тот факт, что $\varphi_{l m}(\vec{r})$ асимптотически стремится к потенциалу соответствующего сферического мультиполя при $r \rightarrow \infty$, придем к равенству (31).

Проведенное рассуждение позволяет решить проблему моментов в электростатике, аналогичную классической проблеме моментов [10]. Допустим, нам известны сферические мультипольные моменты некоторой системы зарядов. Требуется ответить, может ли эта система зарядов находиться в области $V$ и на ее границе $\partial V ?$

Ответ на поставленный вопрос можно получить, переходя с помощью соотношения (30) к характеристическим мультипольным моментам, а затем вычисляя энергию характеристических мультиполей по формуле (17). Если значение энергии окажется конечным, то ответ на поставленный вопрос будет положительным. Соответствуюшее распределение зарядов по $\partial V$ можно будет найти из выражения

$$
\sigma(\vec{r})=\sum_{l=0}^{\infty} \vec{\chi}_{l} \cdot \vec{\sigma}_{l}(\vec{r}) .
$$

Если же значение энергии (17) будет бесконечным, то ответ на поставленный вопрос, очевидно, должен быть отрицательным.

Заметим, что сферические мультипольные моменты для некоторого распределения потенциала можно найти, зная значения потенциала на некоторой сфере с центром в начале координат, в связи с чем проблема моментов предстает как обратная задача электростатики. Она естественно решается с помошью развитого в этой работе аппарата характеристических мультиполей.

\section{7. ПРОВОДНИК ВО ВНЕШНЕМ ЭЛЕКТРОСТАТИЧЕСКОМ ПОЛЕ}

Внешнее электростатическое поле - это поле, создаваемое некоторой фиксированной в пространстве системой зарядов. Энергию проводника во внешнем поле можно определить как работу, необходимую для перемешения проводника из бесконечно удаленной точки в его месторасположение. Эта работа будет равна разности энергий электрического поля зарядов в конечном и начальном положениях. Иначе ее можно найти как полную энергию поля в конечном положении за вычетом собственных энергий внешних зарядов и зарядов проводника, когда он находится в бесконечно удаленной точке, т.е. за вычетом собственной энергии зарядов, распределенных по поверхности проводника с плотностью $q \sigma_{00}(\vec{r})$. Определенная таким образом величина может быть выражена через потенциал внешнего поля $U(\vec{r})$, плотность зарядов $q \sigma_{00}(\vec{r})$ и плотность наведенных внешним полем зарядов (13) по формуле

$$
\begin{gathered}
w=q \int_{\partial V} \sigma_{00}(\vec{r}) U(\vec{r}) d S-\frac{1}{2} \int_{\partial V} \int_{\partial V^{\prime}} U(\vec{r}) K\left(\vec{r}, \vec{r}^{\prime}\right) U\left(\vec{r}^{\prime}\right) d S^{\prime} d S, \\
K\left(\vec{r}, \vec{r}^{\prime}\right)=\sum_{l=1}^{\infty} \sigma_{l}(\vec{r}) \cdot \hat{\alpha}^{(l)} \cdot \sigma_{l}\left(\vec{r}^{\prime}\right) .
\end{gathered}
$$


Первое линейное по потенциалу внешнего поля $U(\vec{r})$ слагаемое здесь соответствует энергии взаимодействия внешнего поля с собственным зарядом проводника, второе же квадратичное по потенциалу внешнего поля слагаемое отвечает энергии взаимодействия наведенных внешним полем зарядов с самим внешним полем. Сушественно, что внешнее поле входит в выражение для $w$ только через свой потенциал $U(\vec{r})$. Интегральный оператор $K\left(\vec{r}, \vec{r}^{\prime}\right)$ в силу положительной определенности матриц $\hat{\alpha}^{(l)}$ будет также неотрицательно определен.

Чтобы $w$ можно было использовать в качестве потенциальной энергии, например, для функции Лагранжа проводника, рассматриваемого как абсолютно твердое тело, следует выразить $w$ через радиус-вектор центра масс проводника $\vec{R}$ и угловые переменные, определяюшие матрицу поворота $\widehat{T}$, совмешаюшего направления осей лабораторной системы координат $\vec{r}$ с осями жестко связанной с твердым телом системы координат $\vec{\xi}$. Это достигается с помошью соотношения

$$
\vec{r}=\vec{R}+\widehat{T} \cdot \vec{\xi}
$$

Обычно при задании матрицы поворота используют углы Эйлера [11]. Сушественно, что при этом зависимости $\sigma_{00}(\vec{r})$ и $K\left(\vec{r}, \vec{r}^{\prime}\right)$ сведутся к зависимостям $\sigma_{00}(\vec{\xi})$ и $K\left(\vec{\xi}, \vec{\xi}^{\prime}\right)$, так что выражение для энергии взаимодействия проводника с внешним электрическим полем можно переписать в форме

$$
\begin{aligned}
w= & q \int_{\partial V} \sigma_{00}(\vec{\xi}) U(\vec{R}+\widehat{T} \cdot \vec{\xi}) d S- \\
& -\frac{1}{2} \int_{\partial V} \int_{\partial V^{\prime}} U\left(\vec{R}+\widehat{T} \cdot \vec{\xi}^{\prime}\right) K\left(\vec{\xi}^{\prime}, \vec{\xi}\right) U(\vec{R}+\widehat{T} \cdot \vec{\xi}) d S^{\prime} d S .
\end{aligned}
$$

Такая форма представления потенциальной энергии проводника весьма удобна для анализа характера точек равновесия проводника во внешнем электростатическом поле. Действительно,

$$
\Delta_{\vec{R}} U(\vec{R}+\widehat{T} \cdot \vec{\xi})=0
$$

т.к. в точках поверхности проводника отсутствуют источники внешнего поля, поэтому нетрудно показать, что

$$
\Delta_{\vec{R}} w=-\int_{\partial V} \int_{\partial V^{\prime}} \nabla_{i} U\left(\vec{R}+\widehat{T} \cdot \vec{\xi}^{\prime}\right) K\left(\vec{\xi}^{\prime}, \vec{\xi}\right) \nabla_{i} U(\vec{R}+\widehat{T} \cdot \vec{\xi}) d S^{\prime} d S \leqslant 0 .
$$

Здесь по повторяюшемуся индексу $i$ предполагается суммирование $(i=x, y, z)$. Функции, удовлетворяющие условию (38), называют супергармоническими функциями. Внутри области супергармоничности эти функции не могут иметь минимумов [12]. Таким образом, равновесие по поступательному перемешению проводника в электростатическом поле может быть только неустойчивым или безразличным, как это имеет место для незаряженного проводника в однородном электрическом поле. 
Нетрудно из соотношения (37) для потенциальной энергии проводника в однородном электрическом поле $\vec{E}_{0}$ получить выражение

$$
w(\vec{R}, \widehat{T})=-q \vec{E}_{0} \cdot(\vec{R}+\widehat{T} \cdot \vec{a})-\frac{1}{2} \vec{E}_{0} \cdot \widehat{T}^{+} \cdot \hat{\alpha} \cdot \widehat{T} \cdot \vec{E}_{0}
$$

где $\hat{\alpha}$ - матрица обычной поляризуемости проводника; $\widehat{T}^{+}-$транспонированная матрица $\widehat{T}$ и

$$
\vec{a}=\int_{\partial V} \sigma_{00}(\vec{\xi}) \vec{\xi} d S_{\vec{\xi}}
$$

- вектор положения в жестко связанной с проводником системе координат центра емкостного распределения заряда $\sigma_{00}(\vec{\xi})$. Из формулы $(39)$, в частности, видно, что действуюший со стороны однородного электрического поля на заряженный проводник момент сил возникает из-за несовпадения центра масс проводника с центром распределения по его поверхности емкостного распределения зарядов, а также из-за отличия тензора поляризуемости проводника $\hat{\alpha}$ от скалярного тензора. Для шара, правильных многогранников и любых других проводников, имеющих две и более осей симметрии порядка выше двух, из (39) будем иметь

$$
w(\vec{R}, \widehat{T})=-q \vec{E}_{0} \cdot \vec{R}-\frac{1}{2} \alpha \vec{E}_{0}^{2},
$$

т.е. получим выражение, не зависящее от угловых переменных, в котором $\alpha$ - скалярная поляризуемость проводника.

В заключение этого раздела отметим, что, строго говоря, энергия взаимодействия проводника с электростатическим полем (37) может быть использована как потенциальная энергия проводника в функции Лагранжа, если при изменении поверхностной плотности зарядов при движении проводника будет отсутствовать диссипация в материале проводника. Таким образом, для сверхпроводников энергию взаимодействия (37) можно рассматривать как потенциальную энергию сверхпроводника, движущегося в электростатическом поле.

\section{8. ЗАКЛЮЧЕНИЕ}

Отметим, что, как свидетельствуют рассмотренные здесь примеры, понятия высших поляризуемостей позволяют не только по-новому взглянуть на известные задачи, но и получать новые результаты - решение проблемы моментов в электростатике и построение функции Лагранжа для сверхпроводника в электростатическом поле. Заметим также, что здесь естественным образом возникает вопрос о характеристических магнитных мультиполях областей пространства, осложняющийся тем, что в литературе отсутствуют понятия даже о сферических магнитных мультиполях. 


\section{Список литературы}

[1] Дж. Джексон. Классическая электродинамика. М.: Мир, 1965.

[2] Д. А. Варшалович, А. Н. Москалев, В. К. Херсонский. Квантовая теория углового момента. Л.: Наука, 1975.

[3] Г. Полиа, Г. Сеге. Изопериметрические неравенства в математической физике. М.: ГИФМЛ, 1962.

[4] В. П. Казанцев, Е. И. Куклин. Радиотехника и электроника. 1989. Т. 35. № 12. С. 2498.

[5] Л. Д. Ландау, Е. М. Лифииц. Электродинамика сплошных сред. М.: Наука, 1982.

[6] Ю.Я. Иоссель, Э.С. Кочанов, М.Г. Струнский. Расчет электрической емкости. Л.: Энергоиздат, 1971.

[7] В. П. Казанцев. Вариационные принципы, характеристические мультиполи и высшие поляризуемости в теории поля. Депонировано в ВИНИТИ, № 2098-В97. М.: ВИНИТИ, 1997.

[8] Н. С. Ланджоф. Основы современной теории потенциала. М.: Наука, 1966.

[9] K. Ректорис. Вариационные методы в математической физике и технике. М.: Мир, 1985.

[10] Н. И. Ахиезер. Классическая проблема моментов. М.: ГИФМЛ, 1961.

[11] Г. Голдстейн. Классическая механика. М.: Наука, 1974.

[12] В. И. Смирнов. Курс высшей математики. Том 4. Часть 2. М.: Наука, 1979.

Поступила в редакцию 11.VI.1998 г., после доработки 17.XI.1998 г. 